\title{
Metastatic Pancreatic Cancer with BRAF and P53 Mutations: Case Report of Therapeutic Response to Doublet Targeted Therapy
}

\author{
Shenthol Sasankan Lorraine Rebuck Gloria Darrah \\ Moises Harari Turquie Ian Rabinowitz \\ Hematology and Medical Oncology, University of New Mexico Comprehensive Cancer \\ Center, Albuquerque, NM, USA
}

\author{
Keywords \\ BRAF mutation · Cancer biology · Gastrointestinal oncology · Pancreatic cancer · Targeted \\ therapy
}

\section{Abstract}

We report on the clinical history of a 49 -year-old female with metastatic pancreatic cancer. She was initially treated with standard chemotherapy as per current guidelines. She was found to have both a BRAF and P53 mutation, and received dabrafenib and trametinib with deep responses, both radiographically and biochemically (CA19-9). Her response has been more clinically relevant than responses in previous case reports of patients with BRAF-positive pancreatic cancer treated with targeted therapy. To the best of our knowledge, this is the first case report showing a dramatic therapeutic response to combination therapy with dabrafenib and trametinib in metastatic pancreatic cancer.

\section{Introduction}

Pancreatic ductal adenocarcinoma (PDAC) is a major cause of cancer-related mortality in the USA, and the seventh leading cause of cancer-related deaths worldwide [1]. In spite of improvements in surgical techniques and new chemotherapy regimens, from 2014 to 2018, the 5-year survival rate for pancreatic cancer has only increased from 6 to $9 \%$. 

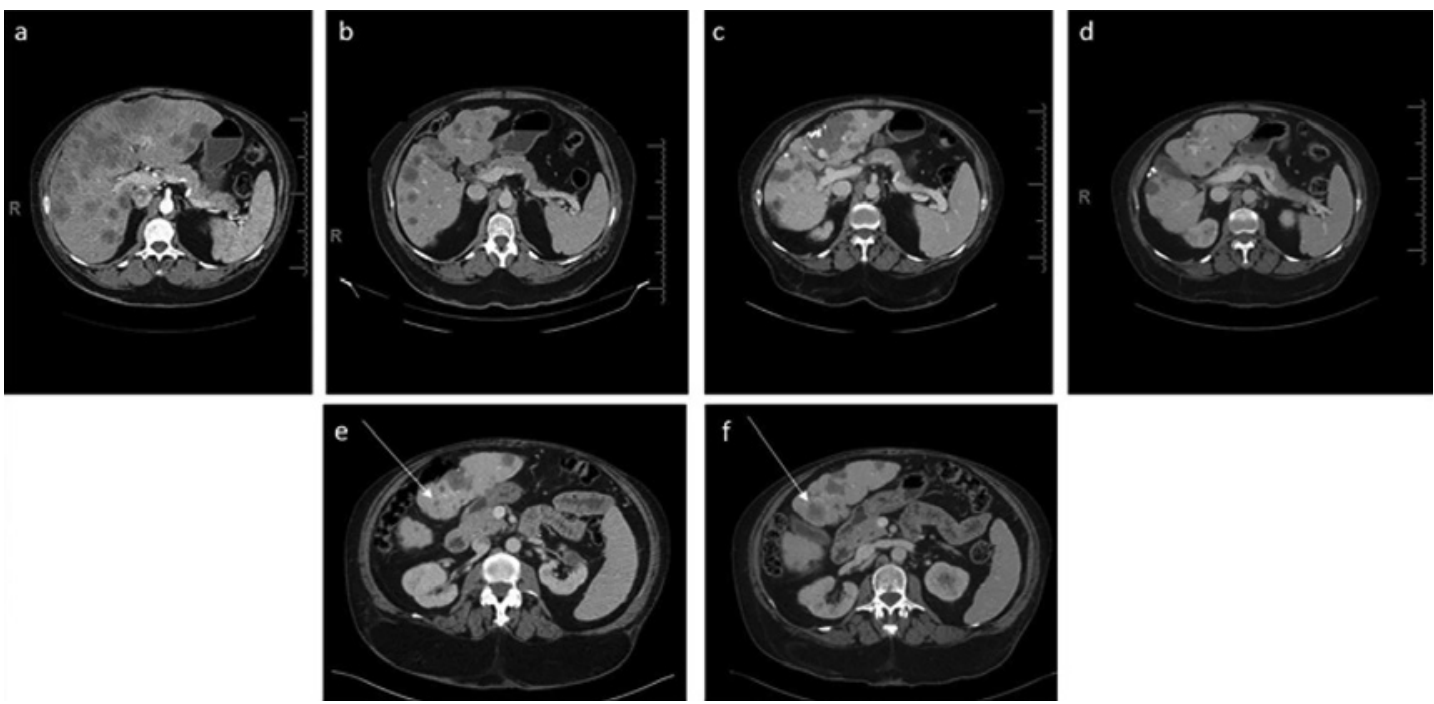

Fig. 1. Serial abdominal contrast CT images of the patient. a At the time of diagnosis - June 2014. b On gemcitabine and Abraxane - October 2015. c On dabrafenib and trametinib with response - September 2019. d On dabrafenib and trametinib with mixed response - March 2020. e Segment 3 lesion in the liver of 0.9 $\mathrm{mm}$ - September 2019.f Single lesion showing an increase in size to $2.2 \mathrm{~cm}$ in segment 3 of the liver - March 2020.

BRAF is mutated in about $15 \%$ of all cancers [2]. The predominant mutation detected in BRAF-mutated cancers is the single substitution of glutamic acid with valine at codon 600 (V600E), representing 70-90\% of all mutations in BRAF [3]. A recently published comprehensive molecular analysis of PDAC by The Cancer Genome Atlas Research Network describes that about 3\% of PDAC tumors harbor the BRAF alteration [4]. Molecularly guided treatments, targeting oncogenic drivers and the DNA damage response and repair pathway, can have a substantial effect on survival in patients with pancreatic cancer [5]. Of note, there are differences in efficacy in treating the same genetic alteration in different tumors [6]. The observation that a significant proportion of melanomas contain BRAF mutations resulted in the development of selective inhibitors of BRAF V600-mutated kinase, namely, vemurafenib and dabrafenib [7]. In a phase III randomized trial, dabrafenib plus trametinib (a MEK inhibitor) significantly improved overall survival among previously untreated patients with metastatic melanoma with BRAF V600E or V600K mutations compared to dabrafenib monotherapy [8]. In a genetically engineered mouse model, the expression of BRAF (V600E) in the mouse pancreas led to pancreatic intraepithelial neoplastic lesions. However, concomitant expression of BRAF (V600E) and TP53 (R270H) results in lethal PDAC [9]. Whether this applies to humans is unclear.

\section{Case Report}

A 49-year-old female presented in April 2014 with abdominal distention and pain. Imaging revealed multiple hepatic lesions, with the largest measuring $9.5 \mathrm{~cm}$, and a heterogeneous mass in the tail of pancreas. She had a biopsy of a liver lesion revealing a moderately differentiated adenocarcinoma consistent with metastatic pancreatic cancer. The CA19-9 level was 51,800 units. The patient was started on gemcitabine and Abraxane, which she tolerated well. Follow-up imaging showed a decreasing tumor burden (Fig. 1) and CA19-9 


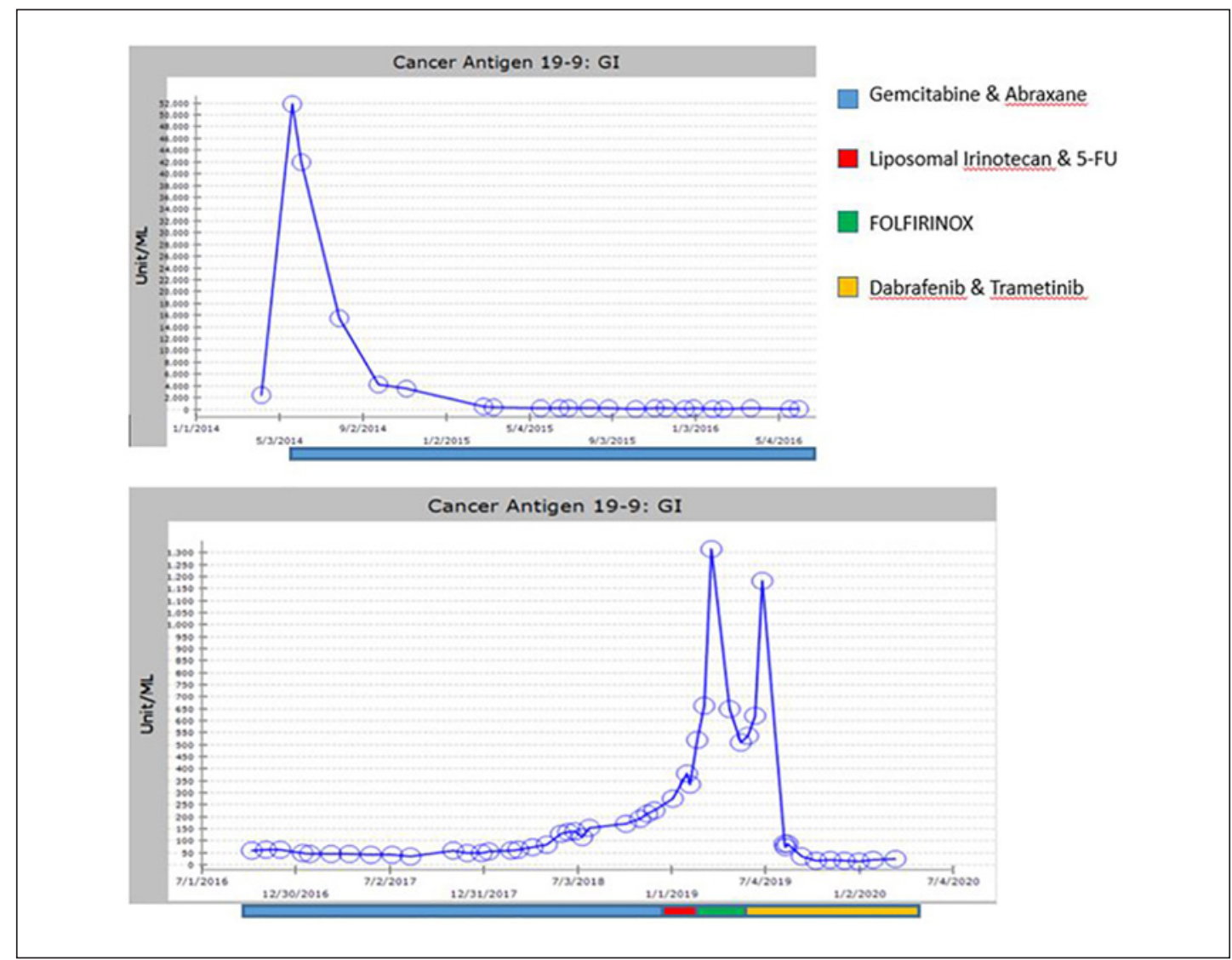

Fig. 2. Cancer antigen 19-9 (CA19-9) marker serum levels (units/mL) over time. Note: both axes are expanded in the second graph.

levels trending down (Fig. 2). In April 2017, there was a chemotherapy dose reduction due to grade 3 thrombocytopenia (caused by cirrhosis and splenomegaly as well as the chemotherapy). By November 2018, the patient's CA19-9 level had begun to increase, and disease progression was confirmed by radiologic imaging. She was then placed on liposomal irinotecan and 5-FU. Her disease continued to progress, as evidenced by an increasing CA19-9 level and radiologic studies performed in February 2019, which showed suspicious pulmonary nodules and an increase in size of metastatic hepatic lesions. She was then started on FOLFIRINOX and her peripheral blood sample was sent for genomic profiling with FoundationOne Liquid in April 2019. The patient was found to have two mutations, BRAF V600E and TP53 C176R. The mutant allele frequency (MAF) of the BRAF and P53 mutations were 1.4 and $0.11 \%$, respectively (Table 1 ).

In June 2019 the patient was noted to have a rapidly rising CA19-9 level, and based on the FoundationOne Liquid results, she was started on dabrafenib $300 \mathrm{mg}$ twice a day with trametinib 2 mg once a day. Shortly after starting the combination, the patient was admitted to the hospital in August 2019 with concern for neutropenic fever and septic shock. Both drugs were held during and after hospitalization. At her next clinic visit, it was noted that her CA19-9 level had significantly dropped, and both drug doses were dropped by $50 \%$. Later the dose of dabrafenib was increased to $300 \mathrm{mg}$ in the morning and $150 \mathrm{mg}$ at night, and trametinib was continued at $2 \mathrm{mg}, 2$ days on and 1 day off. The patient tolerated this dose schedule with minimal side effects. A CT scan in March 2020 revealed that the pulmonary nodules and the 


\section{Case Reports in Oncology}

Table 1. FoundationOne Liquid assay results

\begin{tabular}{l|l}
\hline Case Rep Oncol 2020;13:1239-1243 \\
\hline DOI: 10.1159/000510096 & $\begin{array}{l}\text { @ 2020 The Author(s). Published by S. Karger AG, Basel } \\
\text { www.karger.com/cro }\end{array}$ \\
\hline
\end{tabular}

Sasankan et al.: Metastatic Pancreatic Cancer with BRAF and P53 Mutations

\begin{tabular}{llc}
\hline Gene & Mutation & Mutant allele frequency \\
\hline BRAF & V600E & $1.4 \%$ \\
P53 & C176R & $0.11 \%$ \\
\hline
\end{tabular}

lesion on the tail of the pancreas had disappeared and most liver lesions were either stable or decreased. However, a segment 3 liver lesion had grown from 0.9 to $2 \mathrm{~cm}$ in diameter. This is associated with a slight rise in CA19-9 level. The patient has been referred for stereotactic body radiation therapy in order to ablate this lesion. We plan to continue on the doublet at the current doses, as only a single lesion in the liver had progressed, while the rest of the tumors were stable or shrinking.

\section{Discussion}

To the best of our knowledge, this is the first case report showing a dramatic therapeutic response to combination therapy with dabrafenib and trametinib. There are 4 published reports of BRAF-mutated PDAC patients $(n=8)$ treated with targeted therapy. Five of them were treated with vemurafenib alone, resulting in 4 minimal tumor responses and 1 partial response $[10,11]$. Two of them were treated with a combination of dabrafenib and trametinib, with 1 partial response and 1 minimal response [12]. Our patient had both BRAF and P53 mutations, consistent with the article mentioned above. In another case report, a patient was found to have BRAF, P53, and CDKN2A mutations, and a SMAD4 deletion found on deep sequencing. The patient received single-agent dabrafenib with a good response but relapsed in 4 months and did not respond to the addition of trametinib [13].

Our patient had been on chemotherapy for over 5 years before becoming refractory to chemotherapy, and then was treated with dabrafenib and trametinib. Since she had significant side effects with the doublet at full dose, we dropped her doses accordingly, and she has tolerated the modified doses very well. Her progression-free survival was over 8 months on the two drugs, and she had tumor progression due to a single lesion in the liver. Unfortunately, we were unable to get a biopsy of this lesion to find the molecular basis for progression due to resource constraints. Of interest are the low MAF of both the BRAF and the P53 mutation and the dramatic response to the targeted therapy in our patient. This was most likely due to the FoundationOne Liquid assay underestimating the true MAFs for the two genes.

\section{Conclusion}

This case report highlights the importance of genomic testing in advanced cancers with limited treatment options. Comparing the case reports noted above and our experience, we recommend using both drugs at initiation of therapy, starting the doublet at reduced dosing and then increasing to full dosing as per patient tolerance. Our concern is that patients who are known to carry the BRAF mutation are only treated late in the course of treatment, by which time their poor performance status may preclude them from getting the full benefit of the combined therapy.

\section{Karger'}




\section{Case Reports in Oncology}

\begin{tabular}{l|l}
\hline Case Rep Oncol 2020;13:1239-1243 \\
\hline DOI: 10.1159/000510096 & $\begin{array}{l}\text { @ 2020 The Author(s). Published by S. Karger AG, Basel } \\
\text { www.karger.com/cro }\end{array}$ \\
\hline
\end{tabular}

Sasankan et al.: Metastatic Pancreatic Cancer with BRAF and P53 Mutations

\section{Statement of Ethics}

Informed consent for publication of this case report and associated images was obtained from the patient for this report.

\section{Conflict of Interest Statement}

The authors declare that they have no competing interests.

\section{Funding Sources}

We certify that the authors listed here have no affiliations with or involvement in any organizations and/or any financial interests.

\section{Author Contributions}

S.S. and I.R. conducted the conception and design of the paper and drafted the manuscript. L.R., G.D., and M.H.T. were involved in patient care, manuscript editing, and manuscript review. I.R. was involved in critically revising the manuscript for important intellectual content. All authors read and approved the final manuscript.

\section{References}

1 Bray F, Ferlay J, Soerjomataram I, Siegel RL, Torre LA, Jemal A. Global cancer statistics 2018: GLOBOCAN estimates of incidence and mortality worldwide for 36 cancers in 185 countries. CA Cancer J Clin. 2018;68(6): 394-424.

2 El-Osta H, Falchook G, Tsimberidou A, Hong D, Naing A, Kim K, et al. BRAF mutations in advanced cancers: clinical characteristics and outcomes. PLoS One. 2011:6(10):e25806.

3 Davies H, Bignell GR, Cox C, Stephens P, Edkins S, Clegg S, et al. Mutations of the BRAF gene in human cancer. Nature. 2002;417(6892):949-54.

4 Cancer Genome Atlas Research Network. Integrated genomic characterization of pancreatic ductal adenocarcinoma. Cancer Cell. 2017;32(2):185-203.e13.

5 Pishvaian MJ, Blais EM, Brody JR, Lyons E, DeArbeloa P, Hendifar A, et al. Overall survival in patients with pancreatic cancer receiving matched therapies following molecular profiling: a retrospective analysis of the Know Your Tumor registry trial. Lancet Oncol. 2020;21(4):508-18.

6 Prahallad A, Sun C, Huang S, Di Nicolantonio F, Salazar R, Zecchin D, et al. Unresponsiveness of colon cancer to BRAF(V600E) inhibition through feedback activation of EGFR. Nature. 2012;483(7387):100-3.

7 Menzies AM, Haydu LE, Visintin L, Carlino MS, Howle JR, Thompson JF, et al. Distinguishing clinicopathologic features of patients with V600E and V600K BRAF-mutant metastatic melanoma. Clin Cancer Res. 2012; 18(12):3242-9.

8 Robert C, Karaszewska B, Schachter J, Rutkowski P, Mackiewicz A, Stroiakovski D, et al. Improved overall survival in melanoma with combined dabrafenib and trametinib. N Engl J Med. 2015;372(1):30-9.

9 Collisson EA, Trejo CL, Silva JM, Gu S, Korkola JE, Heiser LM, et al. A central role for RAF $\rightarrow$ MEK $\rightarrow$ ERK signaling in the genesis of pancreatic ductal adenocarcinoma. Cancer Discov. 2012;2(8):685-93.

10 Hainsworth JD, Meric-Bernstam F, Swanton C, Hurwitz H, Spigel DR, Sweeney C, et al. Targeted therapy for advanced solid tumors on the basis of molecular profiles: results from MyPathway, an open-label, phase IIa multiple basket study. J Clin Oncol. 2018 Feb;36(6):536-42.

11 Hyman DM, Puzanov I, Subbiah V, Faris JE, Chau I, Blay JY, et al. Vemurafenib in multiple nonmelanoma cancers with BRAF V600 mutations. N Engl J Med. 2015 Aug;373(8):726-36.

12 Grinshpun A, Zarbiv Y, Roszik J, Subbiah V, Hubert A. Beyond KRAS: practical molecular targets in pancreatic adenocarcinoma. Case Rep Oncol. 2019;12(1):7-13.

13 Wrzeszczynski KO, Rahman S, Frank MO, Arora K, Shah M, Geiger H, et al. Identification of targetable BRAF $\triangle$ N486_P490 variant by whole-genome sequencing leading to dabrafenib-induced remission of a BRAFmutant pancreatic adenocarcinoma. Cold Spring Harb Mol Case Stud. 2019;5(6):a004424. 